\title{
High efficiency broadband ellipse antenna array with tapered parallel-double transmission line feed network
}

\author{
Li Wang ${ }^{1 a)}$, Rui Zhang ${ }^{1}$, Chang-liang Zhao ${ }^{1}$, Yang-tao Wan ${ }^{2}$, \\ Chao $\mathrm{Li}^{1}$, Guang $\mathrm{Fu}^{1}$, and Xiao-wei Shi ${ }^{1}$ \\ ${ }^{1}$ National Laboratory of Antennas and Microwave Technology, Xidian University \\ ${ }^{2}$ Southwest Research Institute of Electronic Equipment, Chengdu, China \\ a)wangkalina@yeah.net
}

\begin{abstract}
In this paper, an antenna element and array for broadband application are proposed. The original antenna element consists of a parallel-double transmission line (PDTL) fed ellipse dipole and a reflecting plate for unidirectional radiation, high-gain, and high efficiency operation. By introducing a circular patch as parasitic radiator, the voltage standing wave ratio (VSWR) $\leq 2$ is achieved from $3 \mathrm{GHz}$ to $9 \mathrm{GHz}$ and the element shows a stable radiation pattern during the operating band. In order to reach a high gain performance, a $4 \times 8$ array is investigated. For the broadband performance of the proposed antenna array, a broadband and low loss tapered PDTL network is used to construct the array. Finally, to verify the feasibility of the proposed antenna, a prototype array is manufactured and measured. Experimental results are found in acceptable agreement with the simulated ones in terms of gain, radiation efficiency, radiation pattern, and VSWR. The result indicates that the proposed antenna has an impedance bandwidth (IBW) for VSWR $\leq 2$ of $100 \%$ ranging from 3.0 to $9.0 \mathrm{GHz}$. Meanwhile the radiation efficiency is better than $82.7 \%$ and the gain varies from 14.8 to $20.9 \mathrm{dBi}$ within the IBW.
\end{abstract}

Keywords: low loss, low profile, broadband array, high efficiency, tapered parallel-double transmission line network

Classification: Microwave and millimeter-wave devices, circuits, and modules

\section{References}

[1] H. Oraizi and R. Pazoki: "Wideband circularly polarized aperture-fed rotated stacked patch antenna," IEEE Trans. Antennas Propag. 61 (2013) 1048 (DOI: 10.1109/TAP.2012.2229378).

[2] L. Li, et al.: "Bandwidth and gain enhancement of patch antenna with stacked parasitic strips based on LTCC technology," Int. J. Antennas Propag. 2014 (2014) 461423 (DOI: 10.1155/2014/461423).

[3] W. Yang, et al.: "Single-fed low profile broadband circularly polarized stacked 
patch antenna,” IEEE Trans. Antennas Propag. 62 (2014) 5406 (DOI: 10.1109/ TAP.2014.2344657).

[4] M. N. Shakib, et al.: "Stacked patch antenna with folded patch feed for ultrawideband application," IET Microw. Antennas Propag. 4 (2010) 1456 (DOI: 10.1049/iet-map.2009.0257).

[5] J. B. Yan, et al.: "A dual-polarized 2-18-GHz Vivaldi array for airborne radar measurements of snow," IEEE Trans. Antennas Propag. 64 (2016) 781 (DOI: 10.1109/TAP.2015.2506734).

[6] H. Liu and M. Sato: "Determination of the phase center position and delay of a Vivaldi antenna," IEICE Electronics Express 10 (2013) 20130573 (DOI: 10.1587/elex.10.20130573).

[7] S. Zhu, et al:: “A compact gain-enhanced Vivaldi antenna array with suppressed mutual coupling for $5 \mathrm{Gmm}$ wave application," IEEE Antennas Wireless Propag. Lett. 17 (2018) 776 (DOI: 10.1109/LAWP.2018.2816038).

[8] E. G. Tianang, et al.: "Ultra-wideband lossless cavity-backed Vivaldi antenna," IEEE Trans. Antennas Propag. 66 (2018) 115 (DOI: 10.1109/TAP.2017. 2775286).

[9] Y. Jia, et al.: "Vivaldi antenna with reduced RCS using half-mode substrate integrated waveguide," IET Electronics Lett. 50 (2014) 345 (DOI: 10.1049/el. 2013.3866).

[10] Y. Dong, et al.: "Vivaldi antenna with pattern diversity for 0.7 to $2.7 \mathrm{GHz}$ cellular band applications," IEEE Antennas Wireless Propag. Lett. 17 (2018) 247 (DOI: 10.1109/LAWP.2017.2783323).

[11] L. Yang, et al.: "Wideband circularly polarised antenna based on tightly coupling effect," IET Electronics Lett. 53 (2017) 448 (DOI: 10.1049/el.2017. 0229).

[12] Y.-J. Hu, et al.: "Broadband circularly polarized microstrip antenna array using sequentially rotated technique," IEEE Antennas Wireless Propag. Lett. 10 (2011) 1358 (DOI: 10.1109/LAWP.2011.2177799).

[13] Z. Y. Zhang, et al.: "Broadband circularly polarized bowtie antenna array using sequentially rotated technique," IEEE Access 6 (2018) 12769 (DOI: 10.1109/ ACCESS.2018.2802938).

[14] L. Zhang, et al.: "Single-feed ultra-wideband circularly polarized antenna with enhanced front-to-back ratio," IEEE Trans. Antennas Propag. 64 (2016) 355 (DOI: 10.1109/TAP.2015.2501844).

[15] V. Sharma: "A novel design of parasitically gap coupled patches forming an elliptical patch antenna for broadband performance,” Chin. J. Eng. 2014 (2014) 365048 (DOI: 10.1155/2014/365048).

[16] D. M. Pozar: Microwave Engineering (Wiley, New York, USA, 2011) 4th ed. 
element to enhance impedance bandwidth, such as stacked antennas $[1,2,3,4]$ and Vivaldi antennas $[5,6,7,8,9,10]$. In [1], the five-layer stacked antenna could reach a broad IBW of $36.15 \%$ for VSWR $\leq 2$. As same as [1], the five-layer stacked parasitic structures were etched at LTCC substrates to enhance the IBW $(16 \%$ for $\left|\mathrm{S}_{11}\right| \leq-10 \mathrm{~dB}$ ) in [2]. However, this kind of stacked antenna suffers from expensive cost and high loss. On account of the broadband performance, Vivaldi antenna can be a good candidate as an element $[5,6,7,8,9,10]$ for antenna array. As reported in [5], the Vivaldi antenna could operate over a $2-18 \mathrm{GHz}$, however, the antenna led to a high profile of $0.7 \lambda$ (where $\lambda$ is the wavelength in free space). In $[7,8]$, the IBW for VSWR $\leq 2$ of the Vivaldi elements are $15 \%$ and $133 \%$, and the heights were $0.97 \lambda$ and $0.56 \lambda$, respectively. Thus, Vivaldi antenna can't be used in some vehicular platforms with low profile requirement. Meanwhile, various wideband feeding networks have been investigated to broaden the IBW of the antenna array. As demonstrated in [11], two-class Wilkinson power divider was utilized to achieve a broad IBW for $\left|\mathrm{S}_{11}\right| \leq-10 \mathrm{~dB}$ of $101 \%$, however, the gain of the antenna array at side frequency was lower than $-5 \mathrm{~dB}$, which revealed a low radiation efficiency. In $[12,13]$, the results illustrated the array IBW was broader than single element, obviously, which means that Wilkinson power divider can ameliorate the bandwidth by sacrifice of the radiation efficiency. Therefore, this type of feeding network is not suitable to preserve the radiation efficiency of antenna array. In summary, to improve the radiation and IBW performance, an antenna element with inherent broadband structure and a low-loss feeding network are the key factors.

In this paper, a broadband array antenna with high efficiency and low profile is presented to satisfy the requirement of vehicle application of remote wireless communication system. The multiple-layer antenna element consists of an ellipse dipole referred from $[14,15]$, a coupled patch and a ground as reflector, which shows a broadband performance in terms of radiation pattern and impedance matching. Then, a broadband tapered PDTL feed network is adopted to a $4 \times 8$ antenna array. Based on tapered PDTL feed network and broadband element, the radiation efficiency and IBW of the array have been crucially enhanced. Finally, a prototype of the proposed antenna array has been fabricated and tested. Measurements were carried out to verify the simulations, and the measured results indicate acceptable agreement with the simulated ones. Consequently, owing to the lowprofile, lightweight, high efficiency and broadband operation, this antenna array can be a good candidate for vehicular application.

\section{Antenna element design}

\subsection{Configuration of the antenna element}

The antenna to be designed is intended to be low profile, lightweight, robust, as simple as possible and have stable radiation performance over a broadband. According to the requirement of remote wireless communication system, the VSWR $\leq 2.5$ operation band should be from $3 \mathrm{GHz}$ to $9 \mathrm{GHz}$. Thus, a broadband planar antenna with PDTL feed has been adopted. Fig. 1 shows the configuration of the antenna element. The proposed antenna structure is composed of three dielectric substrates spaced by air gaps with thicknesses of $h_{2}$ and $h_{4}$, respectively. The gap $h_{4}$ 


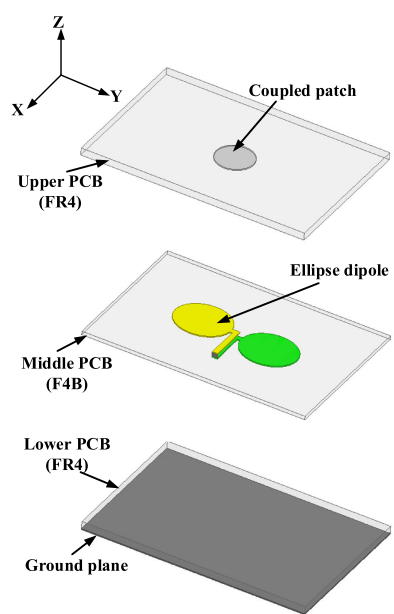

(a)

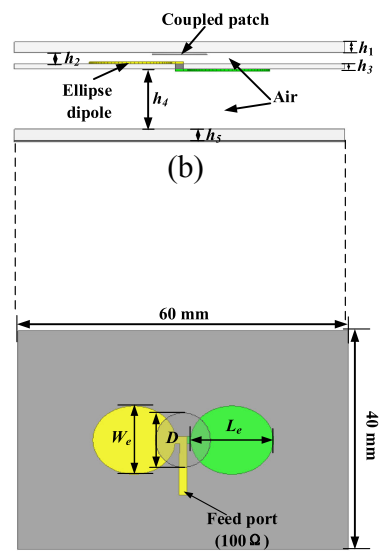

(c)

Fig. 1. Configuration of antenna element. (a) Expanded view. (b) Side view. (c) Top view.

is about $13 \mathrm{~mm}\left(0.065 \lambda_{0}\right.$, where $\lambda_{0}$ is the wavelength at the center frequency), while the $h_{2}$ is equal to $3 \mathrm{~mm}$. Due to the wide IBW, the ellipse dipole, which is printed on a 1-mm-thick substrate F4B with a relative permittivity of $2.65(\tan \delta=0.001)$, is employed instead of conventional dipole. It can be seen that each arm of the dipole is etched on opposite sides of the middle PCB. The top view and the detailed structure of ellipse dipole are shown in Fig. 1(c), while each arm is characterized by a major axis length $L_{\mathrm{e}}$ and a minor axis length $W_{\mathrm{e}}$. The initial $L_{\mathrm{e}}$ was $12.5 \mathrm{~mm}$, which defined as $0.25 \lambda_{0}$ of the center frequency of $6 \mathrm{GHz}$. Due to the impaction of mutual coupling from the coupled patch, the $L_{\mathrm{e}}$ is enlarged to $14.8 \mathrm{~mm}$ to satisfy the desired operating band. In the meantime, $W_{\mathrm{e}}$ is about $12 \mathrm{~mm}$ to reach the broadband operation. As depicted in Fig. 1(a), a tapered PDTL is utilized for the element to achieve a broad band, while the port of the antenna is designed to match with $100 \Omega$. Dielectric substrates of FR4 $(\varepsilon \mathrm{r}=4.4, \tan \delta=0.002)$ with thicknesses of $1 \mathrm{~mm}$ and $2 \mathrm{~mm}$ are selected to construct the upper and lower printed circuit board (PCB). The coupled circular patches are printed on the bottom side of upper PCB. In order to achieve unidirectional radiation, the metal ground is adopted as a reflector and printed on the bottom side of lower PCB. Moreover, the coupled circular patches with a diameter of $D$ can enhance the impedance bandwidth of the antenna, which can be seen in Fig. 2(a). The optimized parameters of the proposed antenna element are listed in Table I.

\subsection{Parametric study}

A simulation model is designed by HFSS.13.0. In order to deeply investigate the proposed antenna, two key parameters of the element are studied and demonstrated in Fig. 2 and 3. These factors include the gap of $h_{2}$ and $h_{4}$. Fig. 2(a) shows the simulated impedance characteristics versus frequency corresponding to different values of $h_{2}$. It can be seen that VSWR at the lower band keeps stable, while the one at middle and the higher band deteriorates with $h_{2}$ increasing. It is obvious that the coupled patch can be treated as a director under this desired structure, the directivity will increase with the gap enlarging. As plotted in Fig. 2(b-c), the 


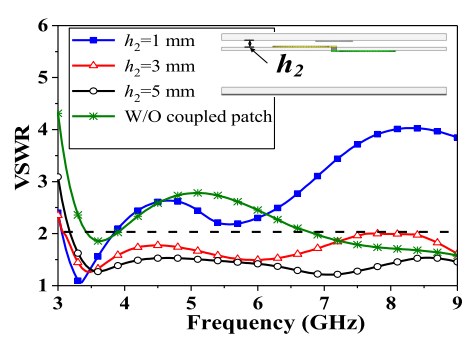

(a)

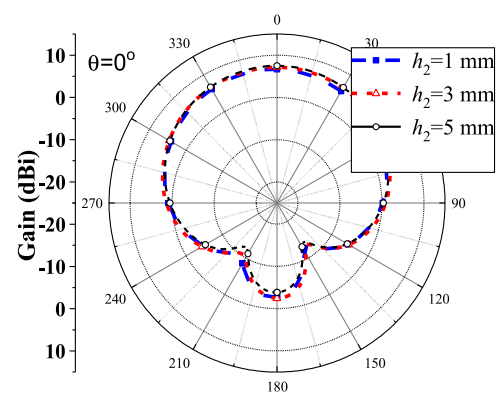

(c)

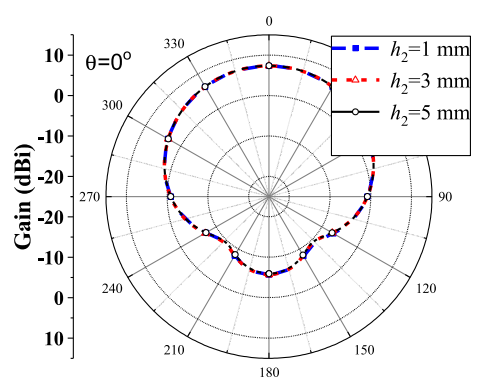

(b)

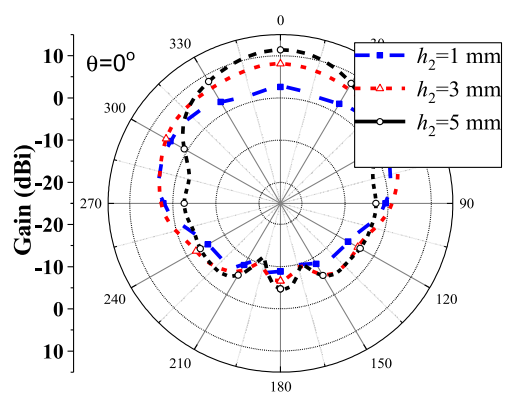

(d)

Fig. 2. Effect of $h_{2}$ on the antenna element. (a) VSWRs. and Gains at (b) $3 \mathrm{GHz}$. (c) $6 \mathrm{GHz}$. (d) $9 \mathrm{GHz}$

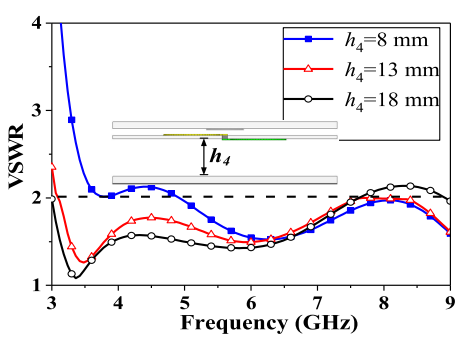

(a)

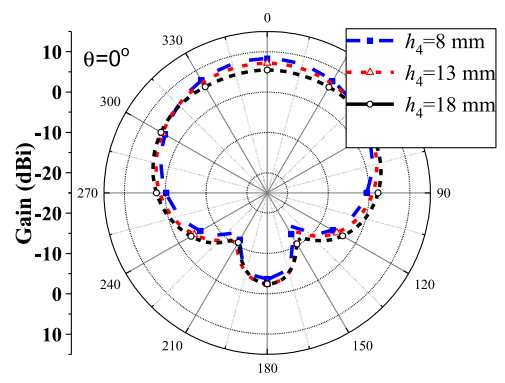

(c)

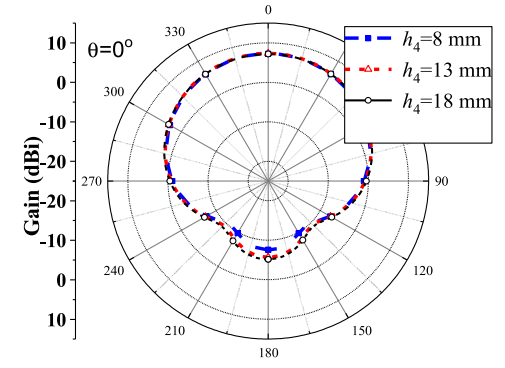

(b)

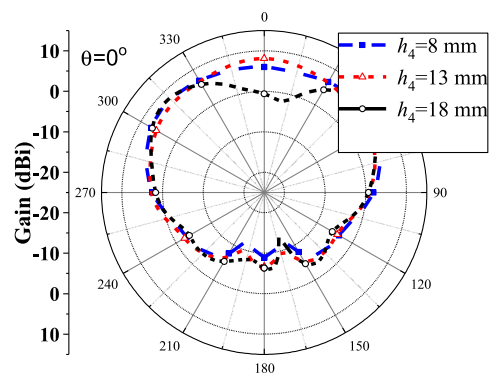

(d)

Fig. 3. Effect of $h_{4}$ on the antenna element. (a) VSWRs. and Gains at (b) $3 \mathrm{GHz}$. (c) $6 \mathrm{GHz}$. (d) $9 \mathrm{GHz}$.

wanted trend is obtained. Due to the difference of electric dimension under a same increment of physical size, the gain at broadside direction increases significantly at $9 \mathrm{GHz}$. Ideally the $h_{2}$ should be as large as possible. However, owing to this tradeoff between the gain, VSWR and low profile, $h_{2}=3 \mathrm{~mm}$ is taken so that the VSWR less than 2 and stable gain patterns over the bandwidth of $3.1-9 \mathrm{GHz}$ is achieved. The gap of $h_{4}$ is also investigated and shown in Fig. 3, from which it is 
found that the VSWR is deteriorating at lower band and getting better at higher band, when the ground becomes further to the middle PCB. Fig. 2(b-c) demonstrates that by varying $h_{4}$, the gain pattern of antenna changes. When $h_{4}$ moves to $18 \mathrm{~mm}$ (almost $0.5 \lambda$ at $9 \mathrm{GHz}$ ), the gain of $9 \mathrm{GHz}$ is depravation dramatically at broadside. The reason is that the reflection wave and the radiation wave overlying in opposite phases at normal side, when the gap turns to $0.5 \lambda$. It is observed that the gain at broadside reaches peak, while $h_{4}$ is equal to $13 \mathrm{~mm}$. In a word, with $h_{2}=3 \mathrm{~mm}$ and $h_{4}=13 \mathrm{~mm}$, the optimal results are obtained for the proposed antenna. From investigation above, it can be seen that these two parameters significantly affect the gain pattern of the antenna at the higher band. Meanwhile, the perfect impedance matching also can be obtained by tuning these gaps.

As shown from the red lines in Fig. 2 and Fig. 3, the peak gains of the proposed antenna element are $7.01 \mathrm{dBi}, 7.11 \mathrm{dBi}$ and $8.09 \mathrm{dBi}$ at 3, 6 and $9 \mathrm{GHz}$, respectively. Meanwhile, the new element shows a broadband impedance for VSWR $\leq 2$ from $3 \mathrm{GHz}$ to $9 \mathrm{GHz}$.

Table I. Dimensions of antenna

\begin{tabular}{c|c|c|c|c|c|c|c|c}
\hline Parameters & $W$ & $W_{1}$ & $L$ & $L_{1}$ & $h_{1}$ & $h_{2}$ & $h_{3}$ & $h_{4}$ \\
\hline Values $(\mathrm{mm})$ & 180 & 20 & 180 & 38 & 2 & 3 & 1 & 13 \\
\hline Parameters & $h_{5}$ & $W_{\mathrm{e}}$ & $L_{\mathrm{e}}$ & $D$ & $W_{\mathrm{s} 1}$ & $W_{\mathrm{s} 2}$ & $L_{\mathrm{s} 1}$ & $L_{\mathrm{s} 2}$ \\
\hline Values $(\mathrm{mm})$ & 1 & 12 & 14.8 & 9.6 & 1.45 & 3 & 15.5 & 24 \\
\hline
\end{tabular}

\section{Antenna array design}

\subsection{Configuration of the antenna array}

In order to maintain the communication distance, the remote wireless communication system requires the peak gain of the proposed array larger than $14 \mathrm{dBi}$ during the operating band. However, only $180 \mathrm{~mm} \times 180 \mathrm{~mm} \times 20 \mathrm{~mm}$ is provided to construct the antenna array by the remote vehicle. Except for the mounting structure, the efficiency area rests only $170 \mathrm{~mm} \times 170 \mathrm{~mm} \times 20 \mathrm{~mm}$ to place the array. According to the equation 1, where $G, A$ and $\eta$ represent the gain, radiation area and radiation efficiency of the antenna, the radiation efficiency should be designed greater than $75 \%$.

$$
\eta=\frac{G \times \lambda^{2}}{4 \pi \times A}
$$

To ensure the stable performance of antenna element, the mutual coupling of each element should be investigated. As depicted in Fig. 4, a $2 \times 2$ subarray is presented to give a suitable layout of antenna array. Fig. 5(a) gives the isolation $\left|\mathrm{S}_{12}\right|$ and VSWR curves of the element with different $W_{1}$, which varies from $33 \mathrm{~mm}$ to $42 \mathrm{~mm}$. From the Fig. 5(a), the distance $W_{1}$ impacts the isolation obviously especially at lower band. The isolation curve becomes lower while the distance becomes wider. On the contrary, the VSWR shows stable performance under the changing of $W_{1}$. When the $L_{1}$ changes, the variation of the isolation and VSWR are given in Fig. 5(b). The isolation rises obviously as the $L_{1}$ increases. It is known that 
the antenna array will keeps stable impedance and radiation property, when the isolation between the antenna elements is bigger than $10 \mathrm{dBi}$. Ideally, the distance between the elements should be as large as possible to achieve high isolation. However, in order to avoid the appearance of grating-lobe and save the space, the $L_{1}=38 \mathrm{~mm}$ and $W_{1}=20 \mathrm{~mm}$ are adopted.

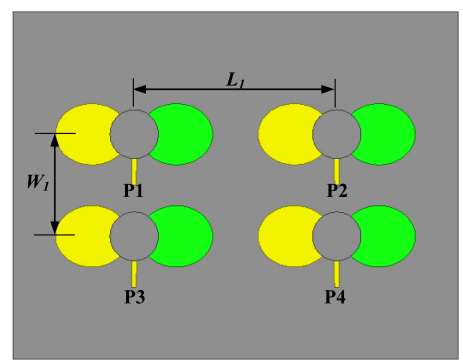

Fig. 4. $2 \times 2$ subarray

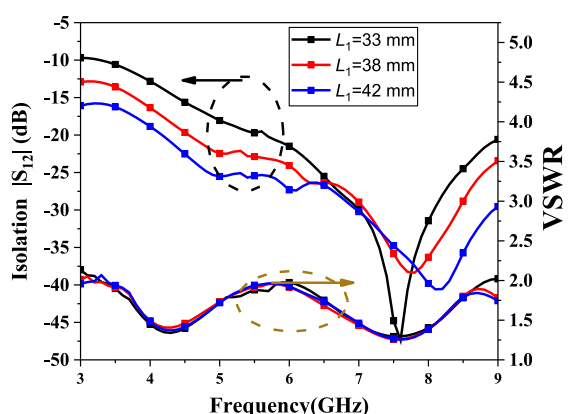

(a)

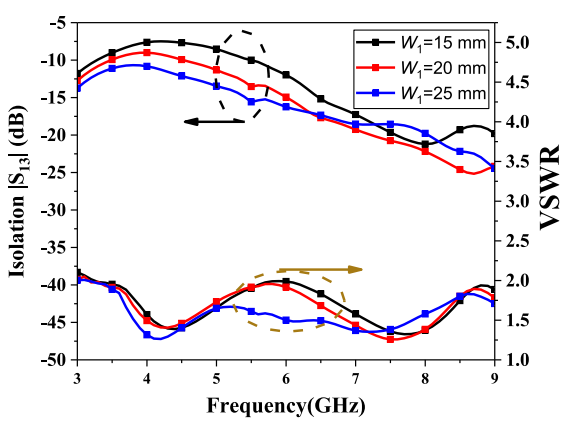

(b)

Fig. 5. Isolations and VSWRs (a) with different $L_{1}$. (b) with different $W_{1}$.

Thus, according to the limited space, the dimension of antenna element and the isolation between the antenna elements, a $4 \times 8$ antenna array with broadband PDTL network is designed and investigated to achieve the goal. Fig. 6 shows the configuration of the antenna. The antenna is composed of five layers which is almost the same with antenna element. All parameters of the substrate are the same as the element in terms of thickness, height, relative permittivity and loss tangent. As displayed in Fig. 6(a), poly tetra fluoroethylene (PTFE) supports are constructed the air layers to enhance the robustness of the antenna. In the meantime, screws are placed through the PTEE to fasten the antenna structure. The top view in Fig. 6(b) exhibits that 4 broadband antenna elements are spaced at $L_{1}$ in Y-axis. Meanwhile, 8 elements are placed in X-axis with gaps of $W_{1}$.

It is known that in [16] the impedance curve of the tapered transmission line shows flat. Compare to the straight line, the tapered transmission line exhibits a broadband impedance character. Thus, a tapered PDTL network has been adopted to this antenna array and the layout are shown in Fig. 6(c). This feed network is etched on the middle PCB and the yellow part is on the top side while the green one is on bottom side. The two parallel dipoles as a subarray whose input impedance is $50 \Omega$, are connected to the output port of the feed network. So that the output port of 


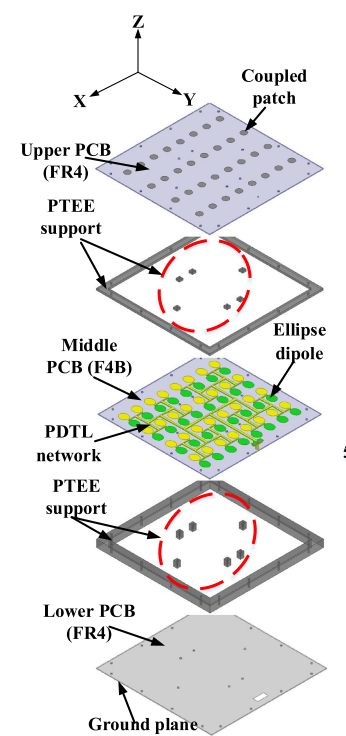

(a)

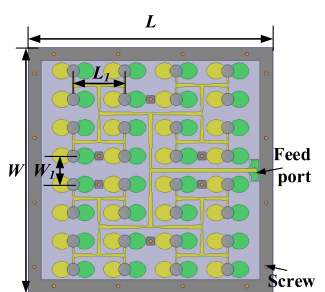

(b)

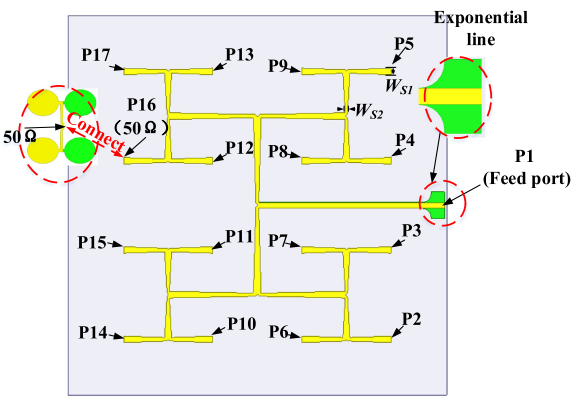

(c)

Fig. 6. Configuration of the proposed antenna. (a) Expanded view. (b) Top view. (c) PDTL network

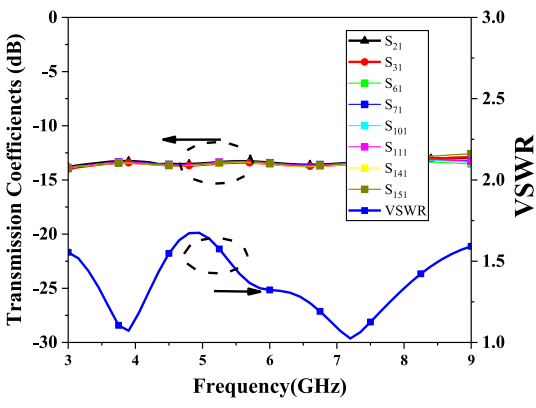

(a)

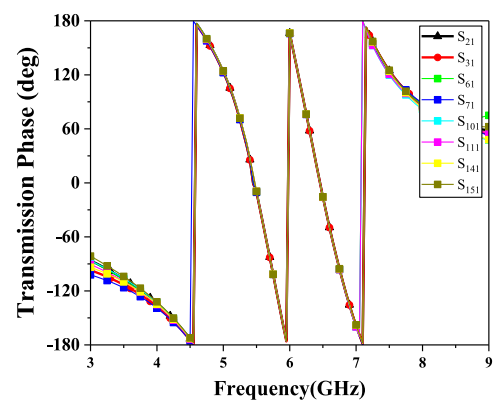

(b)

Fig. 7. Performance of array feed network. (a) Transmission coefficients and VSWR. (b) Transmission phases.

feed network is designed to match with $50 \Omega$. Thus, the element and the feed network make a co-planar structure, which saves space and keeps low-profile. Moreover, the no-resistor network ensures a low-loss of the array, while the tapered PDTL guarantees a broadband impedance performance. In Fig. 7, the simulated results illustrate that the operating band of 3 to $9 \mathrm{GHz}$ for VSWR $\leq 1.5$ is obtained. Due to the symmetry structure, only the S-parameter results of eight output ports are given. During the desired band, the good transmission coefficients and phases maintain almost equal amplitudes and same phases of each port. Consequently, based on the excellent performance of the network, the antenna array could realize stable radiation and impedance characters among a broadband.

\subsection{Measured and simulated results}

The desired antenna array is simulated, fabricated and measured. The geometric optimized parameters are summarized in Table I and the photograph of the fabricated antenna is depicted in Fig. 8. The impedance characteristic, gain and 
radiation pattern are measured by an Agilent E8363B network analyzer and an anechoic chamber. The proposed array has a small size of $180 \mathrm{~mm} \times 180 \mathrm{~mm} \times$ $19 \mathrm{~mm}$ and low-weight of $0.5 \mathrm{~kg}$. The frequency responses of VSWR of the array is plotted in Fig. 9(b). Though some discrepancies between simulated and measured results exist, which are probably caused by assembly tolerances, the experimental result turns out to be acceptable. The experimental result indicates that the ellipse array works well for VSWR $\leq 2$ over $3-9 \mathrm{GHz}$, relatively $100 \%$. The measured radiation efficiency is presented in Fig. 9(a), showing better than $82.7 \%$ within the operating band. The simulated and measured gains of the antenna array are depicted in Fig. 9(a) and agree well with each other, indicating that the gain ranges from 14.8 to $20.9 \mathrm{dBi}$ in the overall band with a peak gain at $9 \mathrm{GHz}$.

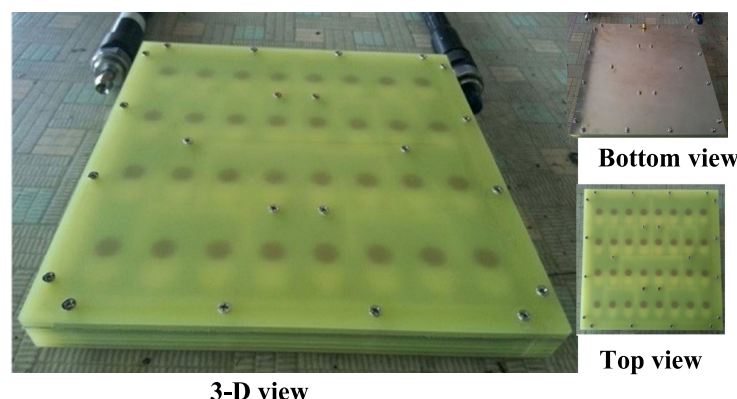

Fig. 8. Photograph of the fabricated array.

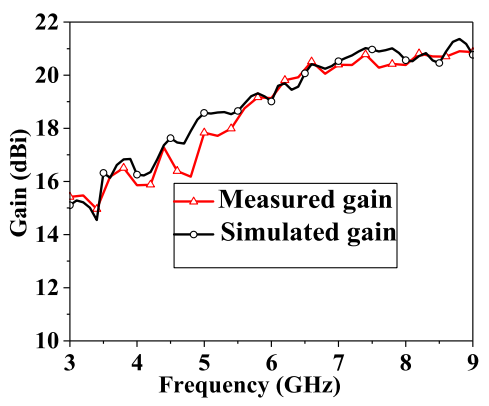

(a)

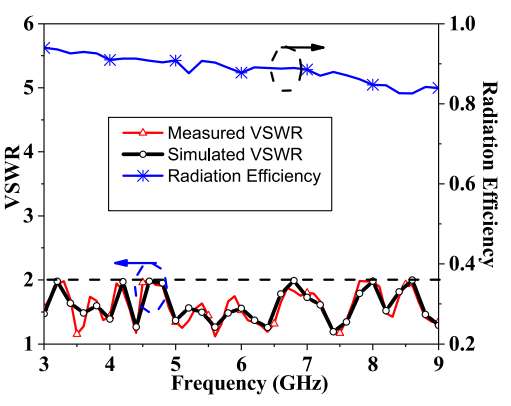

(b)

Fig. 9. Simulated and measured Gains, VSWRs and radiation efficiency of the proposed array.

Fig. 10 depicts the simulated and measured radiation patterns at 3, 6, and $9 \mathrm{GHz}$ in dual plane of XOZ-plane (theta $=0^{\circ}$ ) and YOZ-plane (theta $=90^{\circ}$ ), respectively. There is a perfect agreement between the simulated and measured co-polarization. The results indicate that its half-power beamwidths (HPBWs) are about $28.2^{\circ}, 14.7^{\circ}$, and $9.3^{\circ}$ in XOZ-plane at the frequencies of 3,6 , and $9 \mathrm{GHz}$, respectively, while achieves $29.4^{\circ}, 15.6^{\circ}$, and $10.9^{\circ}$ for the YOZ-plane. Meanwhile, the side-lobes are blow $-10.6 \mathrm{~dB}$ within the plotted frequencies. The measured cross polarizations are also presented, which are less than $20 \mathrm{~dB}$ at the broadside direction. Furthermore, the cross polarization of the measured results are discrepancies with the simulated ones. These differences between the simulated and measured results are expected due to the uncontrollable array fabrication errors, 

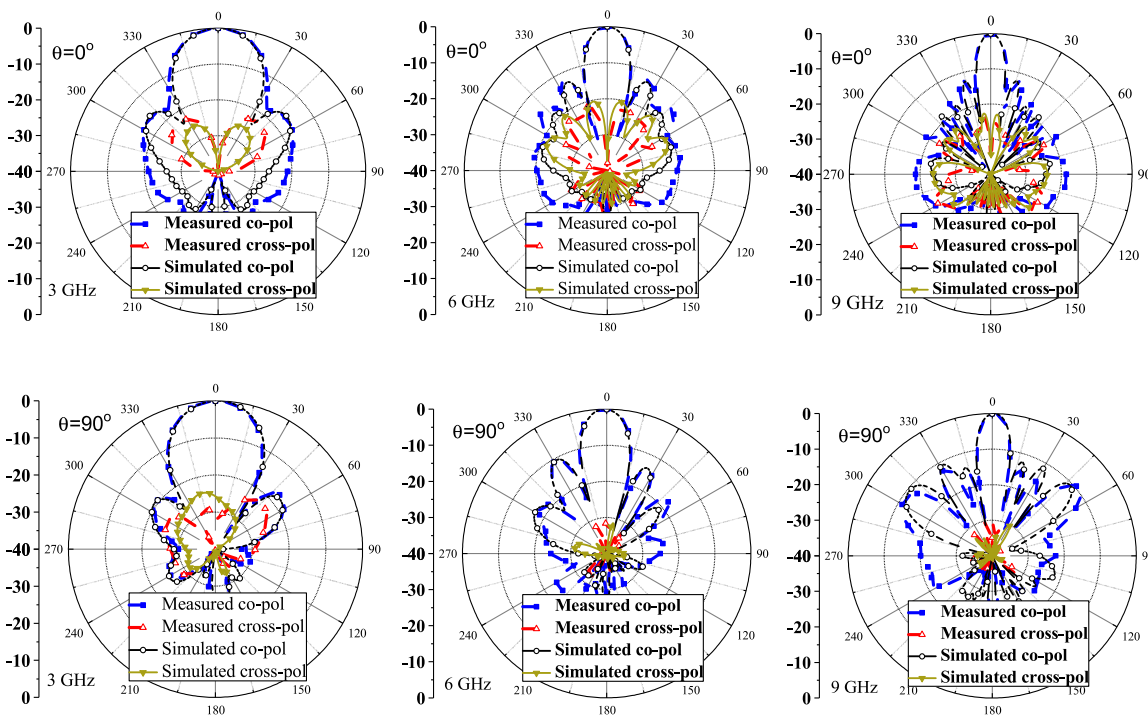

Fig. 10. Simulated and measured radiation patterns of the array at 3,6, and $9 \mathrm{GHz}$.

Table II. Comparison with other published works

\begin{tabular}{c|c|c|c|c}
\hline Ref. & $\begin{array}{c}\text { Bandwidth } \\
(\mathrm{GHz})\end{array}$ & Profile & $\begin{array}{c}\text { Radiation } \\
\text { efficiency }\end{array}$ & $\begin{array}{c}\text { Peak Gain } \\
(\mathrm{dBi})\end{array}$ \\
\hline$[8]$ & $\begin{array}{c}133 \%(1.5-7.5) \\
\text { for VSWR } \leq 2\end{array}$ & $0.56 \lambda$ & not given & 16.5 \\
\hline$[11]$ & $\begin{array}{c}101 \%(1.7-5.2) \\
\text { for }\left|\mathrm{S}_{11}\right| \leq-10 \mathrm{~dB}\end{array}$ & $0.16 \lambda$ & $30 \%-72.3 \%$ & 10.4 \\
\hline$[13]$ & $\begin{array}{c}46 \%(1.1-1.77) \\
\text { for VSWR } \leq 1.5\end{array}$ & $0.24 \lambda$ & $73 \%-83 \%$ & 12.2 \\
\hline $\begin{array}{c}\text { This } \\
\text { work }\end{array}$ & $\begin{array}{c}100 \%(3-9) \\
\text { for VSWR } \leq 2\end{array}$ & $0.19 \lambda$ & $82.7 \%-91.2 \%$ & 20.9 \\
\hline
\end{tabular}

experiment errors, as well as experimental setups that present different conditions from those for the simulations. To validate the good performance of the proposed array, a comparison with other published works on impedance bandwidth, radiation efficiency, profile, and peak gain is carried out in Table II.

In addition, the array antenna exhibits broadband impedance performance with stable radiation pattern and high radiation efficiency.

\section{Conclusions}

In this paper, a broadband antenna array with high efficiency is presented. The antenna element introduces a broadband ellipse dipole and tapered feed line to obtain broad IBW. Then, a $4 \times 8$ ellipse array is investigated. By employing a broadband tapered PDTL feed network, the proposed array achieves high efficiency performance during a broadband. Furthermore, due to the co-planar structure, a simple configuration and low-profile are obtained. Finally, an array prototype is fabricated and tested. The results show acceptable agreement between simulation and testation. The IBW of $100 \%(3-9 \mathrm{GHz})$ for VSWR $\leq 2$ is achieved. The 
measured gain varies from 14.8 to $20.9 \mathrm{dBi}$ and the radiation efficiency keeps above $82.7 \%$ in overall band. In brief, due to the low-profile, lightweight and broadband high performance, the proposed array could be a good candidate for the vehicular application of remote wireless communication system.

\section{Acknowledgements}

The authors would like to thank Professor Shuxi Gong for valuable suggestions. 